\title{
Tractability of approximating multivariate linear functionals
}

\author{
Erich Novak and Henryk Woźniakowski
}

To Stephen Smale on the occasion of his 80th birthday

\begin{abstract}
We review selected tractability results for approximating linear tensor product functionals defined over reproducing kernel Hilbert spaces. This review is based on Volume II of our book Tractability of Multivariate Problems. In particular, we show that all nontrivial linear tensor product functionals defined over a standard tensor product unweighted Sobolev space suffer the curse of dimensionality and therefore they are intractable. To vanquish the curse of dimensionality we need to consider weighted spaces, in which all groups of variables are monitored by weights. We restrict ourselves to product weights and provide necessary and sufficient conditions on these weights to obtain various kinds of tractability.
\end{abstract}

Mathematics Subject Classification (2010). 65D15, 65D30, 65Y20.

Keywords. Tractability, curse of dimensionality, weighted spaces, numerical integration, tensor product Sobolev spaces.

\section{Introduction}

Tractability of multivariate problems has recently been a popular research subject. Such problems are defined on spaces of $d$-variate functions, where $d$ can be arbitrarily large. We want to compute an $\varepsilon$-approximation of the $d$-variate multivariate problem by algorithms that use finitely many function values. The minimal number $n(\varepsilon, d)$ of function values needed for computation of an $\varepsilon$-approximation for the $d$-variate case is a good measure of the computational complexity. The minimal number $n(\varepsilon, d)$ has been usually studied for a fixed $d$ and for $\varepsilon$ tending to zero. Tractability studies $n(\varepsilon, d)$ as a function of two variables, hoping to reveal conditions under which $n(\varepsilon, d)$ is not exponential neither in $\varepsilon^{-1}$ nor in $d$. Such studies go back about 15 years, see $[10,11]$. 
There are various kinds of tractability, depending on how we define the lack of exponential dependence. The first papers were devoted to polynomial tractability, in which $n(\varepsilon, d)$ is bounded by a polynomial in $\varepsilon^{-1}$ and $d$, so that there are three nonnegative numbers $C, q$ and $p$ such that

$$
n(\varepsilon, d) \leq C d^{q} \varepsilon^{-p} \quad \text { for all } \varepsilon \in(0,1) \text { and } d \in \mathbb{N} .
$$

If $q=0$, then we say that the problem is strongly polynomially tractable, and in this case the infimum of $p$ satisfying (1) is called the exponent of strong polynomial tractability.

Weak tractability is defined when

$$
\lim _{\varepsilon^{-1}+d \rightarrow \infty} \frac{\ln n(\varepsilon, d)}{\varepsilon^{-1}+d}=0 .
$$

Weak tractability means that

$$
n(\varepsilon, d)=\exp \left(o\left(\varepsilon^{-1}+d\right)\right)
$$

cannot go exponentially fast to infinity with either $\varepsilon^{-1}$ or $d$. However, it may go to infinity faster than polynomially in $\varepsilon^{-1}$ and $d$. For example, if

$$
n(\varepsilon, d)=\Theta\left(\exp \left(\sqrt{\varepsilon^{-1}+d}\right)\right),
$$

then we do not have polynomial tractability, but weak tractability holds.

If $n(\varepsilon, d)$ goes to infinity exponentially fast with $d$, then we say that the problem suffers the curse of dimensionality and is intractable. There are other kinds of tractability, but in this paper we restrict ourselves only to (strong) polynomial and weak tractability.

Tractability can be studied in various settings such as the worst case, average case, probabilistic and randomized settings. The definition of an $\varepsilon$ approximation can also vary and include the absolute, normalized and relative error criteria. Here we will consider only the worst case setting for the normalized error criterion.

The current state of tractability research is presented in our book Tractability of Multivariate Problems. The first volume [4] mostly studies tractability when we can use more general information than function values, which are specified by arbitrary linear functionals. Such information is reasonable for approximating linear operators with infinite- (or very high-) dimensional target spaces, or for nonlinear operators with arbitrary target spaces. The second volume [5] mostly studies tractability of linear functionals for algorithms using function values. The third volume [6] will be devoted to tractability study of mostly linear operators for algorithms using function values, and hopefully will be finished in a year or two.

The purpose of this paper is to present a sample of tractability results. Therefore we have decided to restrict ourselves to tractability of linear functionals based on [5]. We want to stress that tractability results for linear functionals are very rich in possibilities and that almost anything can happen. Indeed, for some infinite-dimensional reproducing kernel Hilbert spaces, all linear functionals are trivial and can be approximated with arbitrarily 
small worst case error by using just one function value. The first construction of such a space was done in [2] and also can be found in Chapter 10 of [5]. On the other hand, linear functionals can be very hard. Indeed, there are Hilbert reproducing kernel spaces for which all nontrivial linear tensor product functionals suffer from the curse of dimensionality. We show such an example for a standard tensor product unweighted Sobolev space in Section 2.

The negative tractability results usually hold for unweighted spaces. In this case, all variables and groups of variables play the same role. This is the main reason for the exponential dependence on $d$. To vanquish the curse of dimensionality we study weighted spaces, in which the role of variables and groups of variables is monitored by weights. We can model problems for which the dependence on the successive variables or groups of variables is diminishing by decaying weights. This sometimes allows us to obtain (strong) polynomial or weak tractability.

There are many types of weights, such as product, finite-order, finitediameter and order-dependent weights. Again we restrict ourselves in this paper only to product weights, which were introduced in [7]. Product weights were the first ones for which a tractability study was performed. In Section 3 we give conditions on product weights and on linear tensor product functionals that yield (strong) polynomial or weak tractability.

We hope that the readers will get a general idea of tractability study. Much more can be found in many tractability papers that are, in particular, mentioned in Volumes I and II of $[4,5]$.

\section{Tensor product Sobolev space}

For $d=1$, consider the Sobolev space $H_{1}$ of real functions $f:[0,1] \rightarrow \mathbb{R}$ that are absolutely continuous and whose first derivatives are square integrable. The inner product in $H_{1}$ is given by

$$
\langle f, g\rangle_{H_{1}}=f(0) g(0)+\int_{0}^{1} f^{\prime}(t) g^{\prime}(t) \mathrm{d} t \quad \text { for all } f, g \in H_{1} .
$$

It is well known that $H_{1}$ is a reproducing kernel Hilbert space with the kernel

$$
K_{1}(x, t)=1+\min (x, t) \text { for all } x, t \in[0,1] .
$$

For $d \geq 1$, we take the $d$-fold tensor product of $H_{1}$ and obtain

$$
H_{d}=H_{1} \otimes H_{1} \otimes \cdots \otimes H_{1} .
$$

The Sobolev space $H_{d}$ is the space of real functions defined on $[0,1]^{d}$ with the inner product

$$
\begin{array}{r}
\langle f, g\rangle_{H_{d}}=f(0) g(0)+\sum_{\emptyset \neq \mathfrak{u} \subseteq\{1,2, \ldots, d\}} \int_{[0,1]^{|\mathfrak{u}|}} \frac{\partial^{|\mathfrak{u}|} f}{\partial x_{\mathfrak{u}}}\left(x_{\mathfrak{u}}, 0\right) \frac{\partial^{|\mathfrak{u}|} g}{\partial x_{\mathfrak{u}}}\left(x_{\mathfrak{u}}, 0\right) \mathrm{d} x_{\mathfrak{u}} \\
\quad \text { for all } f, g \in H_{d} .
\end{array}
$$


Here, $y=\left(x_{\mathfrak{u}}, 0\right)$ denotes a $d$-dimensional vector with components $y_{j}=x_{j}$ for $j \in \mathfrak{u}$ and $y_{j}=0$ for $j \notin \mathfrak{u}$. Obviously, $|\mathfrak{u}|$ is the cardinality of the set $\mathfrak{u}$, and $\partial x_{\mathfrak{u}}$ stands for $\prod_{j \in \mathfrak{u}} \partial x_{j}$.

The space $H_{d}$ is also a reproducing kernel space with the kernel

$$
K_{d}(x, t)=\prod_{j=1}^{d}\left(1+\min \left(x_{j}, t_{j}\right)\right) \quad \text { for all } x, t \in[0,1]^{d} .
$$

We consider a linear tensor product functional $I_{d}: H_{d} \rightarrow \mathbb{R}$ given by

$$
I_{d}(f)=\left\langle f, h_{d}\right\rangle_{H_{d}} \quad \text { for all } f \in H_{d},
$$

where

$$
h_{d}(x)=\prod_{j=1}^{d} h_{1}\left(x_{j}\right) \quad \text { for all } x \in[0,1]^{d} .
$$

Here, $h_{1} \in H_{1}$, and obviously $h_{d} \in H_{d}$. We also have

$$
\left\|I_{d}\right\|=\left\|h_{d}\right\|_{H_{d}}=\left\|h_{1}\right\|_{H_{1}}^{d} .
$$

We approximate $I_{d}$ by algorithms $A_{n}$ using $n$ function values. Without loss of generality, we may assume that $A_{n}$ is linear, so that

$$
A_{n}(f)=\sum_{j=1}^{n} a_{j} f\left(t_{j}\right) \quad \text { for all } f \in H_{d}
$$

for some $a_{j} \in \mathbb{R}$ and some sample points $t_{j} \in[0,1]^{d}$. The worst case error of $A_{n}$ is defined as

$$
e^{\mathrm{wor}}\left(A_{n}\right):=\sup _{\|f\|_{H_{d}} \leq 1}\left|I_{d}(f)-A_{n}(f)\right|=\left\|I_{d}-A_{n}\right\| .
$$

For $n=0$ we set $A_{0}=0$, and then the worst case error

$$
e^{\text {wor }}(0)=\left\|I_{d}\right\|
$$

is the initial error that can be achieved without sampling the function $f$.

We want to improve the initial error by a factor $\varepsilon \in(0,1)$, and this corresponds to the normalized error criterion. Let

$$
n\left(\varepsilon, I_{d}\right)=\min \left\{n \mid \exists A_{n} \text { such that } e^{\text {wor }}\left(A_{n}\right) \leq \varepsilon e^{\text {wor }}(0)\right\}
$$

be the minimal number of function values that is necessary to reduce the initial error by a factor $\varepsilon$. The minimal number $n\left(\varepsilon, I_{d}\right)$ is called the information complexity, and it is almost the same as the computational complexity (minimal cost) of solving the problem $I_{d}$ to within $\varepsilon$.

Let $I=\left\{I_{d}\right\}$ be the linear tensor product problem, or shortly the problem. We say that the problem $I$ is (strongly) polynomially tractable if (1) holds, and the problem $I$ is weakly tractable if (2) holds.

It is natural to ask, for which problems $I$ we have (strong) polynomial or weak tractability. Observe that the problem $I$ is fully determined by $h_{1}$, which is a representer of $I_{1}$ for the univariate case. Obviously, for some $h_{1}$ the 
problem $I$ is trivial. Indeed, if $h_{1}=0$, then $I_{d}=0$ for all $d$ and $n(\varepsilon, d)=0$. More generally, if

$$
h_{1}(x)=a K_{1}(x, t)=a[1+\min (x, t)] \text { for all } x \in[0,1],
$$

for some $a \in \mathbb{R}$ and some $t \in[0,1]$, then

$$
I_{1}(f)=\left\langle f, h_{1}\right\rangle_{H_{1}}=a\left\langle f, K_{1}(\cdot, t)\right\rangle_{H_{1}}=a f(t) \quad \text { for all } f \in H_{1} .
$$

Furthermore, $h_{d}(x)=a^{d} \prod_{j=1}^{d}\left[1+\min \left(x_{j}, t\right)\right]=a^{d} K_{d}(x,[t, t, \ldots, t])$, and therefore

$$
I_{d}(f)=a^{d}\left\langle f, K_{d}(\cdot,[t, t, \ldots, t])\right\rangle_{H_{d}}=a^{d} f(t, t, \ldots, t) \quad \text { for all } f \in H_{d} .
$$

Then the algorithm $A_{1}$ with $a_{1}=a^{d}$ and $t_{1}=[t, t, \ldots, t]$ has the form

$$
A_{1}(f)=a_{1} f\left(t_{1}\right)=a^{d} f(t, t, \ldots, t)=I_{d}(f),
$$

and its worst case error is zero. Hence, $n(\varepsilon, d) \leq 1$. More precisely, $n(\varepsilon, d)=0$ if $a=0$ and otherwise $n(\varepsilon, d)=1$. In either case, the problem $I$ is trivial and we have strong polynomial tractability with the exponent 0 .

We say that the tensor product problem $I$ is nontrivial if (3) does not hold for any $a \in \mathbb{R}$ and $t \in[0,1]$. We now ask whether there are any nontrivial problems that are (strongly) polynomial tractable or weakly tractable. The following theorem answers this question in the negative.

Theorem 1. All nontrivial linear tensor product problems suffer from the curse of dimensionality. More precisely, for all such problems I there exist $C>1$ and $\varepsilon_{0} \in(0,1)$ depending on $h_{1}$ such that

$$
n\left(\varepsilon, I_{d}\right) \geq C^{d} \quad \text { for all } \varepsilon \in\left(0, \varepsilon_{0}\right) \text { and } d \in \mathbb{N} .
$$

Proof. This theorem is a special case of Theorem 11.15 in Chapter 11, see also Example 11.6.1, of [5]. The proof is based on the fact that the reproducing kernel $K_{1}$ has a so-called decomposable part and uses the proof technique developed in [3].

What can we do with this negative result? We can claim that the Sobolev space $H_{d}$ is too large for large $d$. Indeed, note that all variables and groups of variables in $H_{d}$ play exactly the same role. That is, for $f \in H_{d}$ define

$$
g\left(t_{1}, t_{2}, \ldots, t_{d}\right)=f\left(t_{j_{1}}, t_{j_{2}}, \ldots, t_{j_{d}}\right) \text { for all } t_{j} \in[0,1]
$$

for an arbitrary permutation of $\left(j_{1}, j_{2}, \ldots, j_{d}\right)$ of $(1,2, \ldots, d)$. Then

$$
g \in H_{d} \quad \text { and } \quad\|g\|_{H_{d}}=\|f\|_{H_{d}} .
$$

Perhaps the curse of dimensionality will disappear if all variables and groups of variables play a different role and are monitored by weights. This is the subject of our next section. 


\section{Weighted tensor product Sobolev space}

It seems natural to shrink the Sobolev space by demanding that some variables and groups of variables are more important than others. This can be accomplished by introducing weights that monitor the importance of all variables and groups of variables. To simplify our presentation we restrict ourselves only to product weights, referring the reader to $[4,5]$ for general weights.

For product weights, we redefine the reproducing kernel as

$$
K_{d, \gamma}(x, t)=\prod_{j=1}^{d}\left(1+\gamma_{j} \min \left(x_{j}, t_{j}\right)\right) \quad \text { for all } x, t \in[0,1]^{d} .
$$

Here, $\gamma=\left\{\gamma_{j}\right\}$ and all $\gamma_{j}$ 's are positive and ordered,

$$
1=\gamma_{1} \geq \gamma_{2} \geq \cdots \geq \gamma_{d} \geq \cdots>0 .
$$

An example of such product weights is given by letting $\gamma_{j}=j^{-\beta}$ for $\beta \geq 0$.

Let $H_{d, \gamma}=H\left(K_{d, \gamma}\right)$ be the modified Sobolev space. The inner product in $H_{d, \gamma}$ is now given by

$$
\begin{aligned}
\langle f, g\rangle_{H_{d, \beta}}= & f(0) g(0)+\sum_{\emptyset \neq \mathfrak{u} \subseteq\{1,2, \ldots, d\}} \prod_{j \in \mathfrak{u}} \gamma_{j}^{-1} \\
& \times \int_{[0,1]^{|\mathfrak{u}|}} \frac{\partial^{|\mathfrak{u}|} f}{\partial x_{\mathfrak{u}}}\left(x_{\mathfrak{u}}, 0\right) \frac{\partial^{|\mathfrak{u}|} g}{\partial x_{\mathfrak{u}}}\left(x_{\mathfrak{u}}, 0\right) \mathrm{d} x_{\mathfrak{u}} \quad \text { for all } f, g \in H_{d} .
\end{aligned}
$$

Note that algebraically the spaces $H_{d, \gamma}$ and $H_{d}$ are the same and

$$
\|f\|_{H_{d}} \leq\|f\|_{H_{d, \gamma}} \leq \frac{1}{\prod_{j=1}^{d} \gamma_{j}^{1 / 2}}\|f\|_{H_{d}} \quad \text { for all } f \in H_{d} .
$$

This implies that the linear tensor product functionals $I_{d}(f)=\left\langle f, h_{d}\right\rangle_{H_{d}}$ are also well defined over $H_{d, \gamma}$. To stress the new domain $H_{d, \gamma}$ of $I_{d}$ we denote

$$
I_{d, \gamma}(f)=I_{d}(f) \text { for all } f \in H_{d, \gamma} .
$$

Note that we now have $I_{d, \gamma}(f)=\left\langle f, h_{d, \gamma}\right\rangle_{H_{d, \gamma}}$ with

$$
h_{d, \gamma}(x)=\prod_{j=1}^{d}\left[h_{1}(0)+\gamma_{j}\left(h_{1}\left(x_{j}\right)-h_{1}(0)\right)\right] \quad \text { for all } x \in[0,1]^{d}
$$

and

$$
\left\|I_{d, \beta}\right\|=\left\|h_{d, \gamma}\right\|_{H_{d, \gamma}}=\prod_{j=1}^{d}\left(h_{1}^{2}(0)+\gamma_{j} \int_{0}^{1}\left[h_{1}^{\prime}(t)\right]^{2} \mathrm{~d} t\right)^{1 / 2} .
$$
Then

To understand the role of the weights, take $f \in H_{d, \gamma}$ with $\|f\|_{H_{d, \gamma}}=1$.

$$
\int_{[0,1]^{|\mathfrak{u}|}}\left(\frac{\partial^{|\mathfrak{u}|} f}{\partial x_{\mathfrak{u}}}\left(x_{\mathfrak{u}}, 0\right)\right)^{2} \mathrm{~d} x_{\mathfrak{u}} \leq \prod_{j \in \mathfrak{u}} \gamma_{j} .
$$

Assume for a moment that $\lim _{j} \gamma_{j}=0$. Then for $\mathfrak{u}$ with a large cardinality, the corresponding partial derivatives are small in the $L_{2}$ sense. If the $\gamma_{j}$ 's go 
to zero, then all partial derivatives go to zero and the space $H_{d, \gamma}$ becomes the space of constant functions.

One may hope that the curse of dimensionality will be vanquished for product weights that decay sufficiently quickly. As we will see, this hope will be only partially fulfilled, at the expense of some additional assumptions on $h_{1}$.

The first troublesome case is when $h_{1}(0)=0$. Then

$$
h_{d, \gamma}=\left[\prod_{j=1}^{d} \gamma_{j}\right] h_{d}
$$

Note that the only trivial problem with this property is when $h_{1}=0$. For $h_{1}(0)=0$ and $h_{1} \neq 0$, the initial error (4) becomes

$$
\left\|I_{d, \gamma}\right\|=\left[\prod_{j=1}^{d} \gamma_{j}^{1 / 2}\right] \cdot\left[\int_{0}^{1}\left[h_{1}^{\prime}(t)\right]^{2} \mathrm{~d} t\right]^{d / 2}=\left[\prod_{j=1}^{d} \gamma_{j}^{1 / 2}\right] \cdot\left\|h_{d}\right\|_{H_{d}}>0 .
$$

We now show that for such problems we still have the curse of dimensionality independently of the product weights.

Theorem 2. Let $I_{\gamma}=\left\{I_{d, \gamma}\right\}$ be a linear tensor product problem defined over the weighted Sobolev space $H_{d, \gamma}$ with

$$
h_{1}(0)=0 \quad \text { and } \quad h_{1} \neq 0 .
$$

Then for arbitrary positive product weights, the problem $I_{\gamma}$ suffers from the curse of dimensionality. More precisely, there exist $C>1$ and $\varepsilon_{0} \in(0,1)$ depending on $h_{1}$ and independent of the weights $\gamma$ such that

$$
n\left(\varepsilon, I_{d, \gamma}\right) \geq C^{d} \quad \text { for all } \varepsilon \in\left(0, \varepsilon_{0}\right) \text { and } d \in \mathbb{N} \text {. }
$$

Proof. Take an arbitrary linear algorithm $A_{n}(f)=\sum_{j=1}^{n} a_{j} f\left(t_{j}\right)$ for $f \in$ $H_{d, \gamma}$. It is well known that the square of the worst case of $A_{n}$ is given by

$$
\begin{aligned}
& {\left[e^{\text {wor }}\left(A_{n}\right)\right]^{2}} \\
& \quad=\left\|h_{d, \gamma}\right\|_{H_{d, \gamma}}^{2}-2 \sum_{j=1}^{n} a_{j} h_{d, \gamma}\left(t_{j}\right)+\sum_{i, j=1}^{n} a_{i} a_{j} K_{d, \gamma}\left(t_{i}, t_{j}\right) \\
& \quad=\left[\prod_{j=1}^{d} \gamma_{j}\right]\left(\left\|h_{d}\right\|_{H_{d}}^{2}-2 \sum_{j=1}^{n} a_{j} h_{d}\left(t_{j}\right)+\frac{1}{\prod_{j=1}^{d} \gamma_{j}} \sum_{i, j=1}^{n} a_{i} a_{j} K_{d, \gamma}\left(t_{i}, t_{j}\right)\right) .
\end{aligned}
$$

We have

$$
K_{d, \gamma}(x, y)=\sum_{\mathfrak{u} \subseteq\{1,2, \ldots, d\}}\left[\prod_{j \in \mathfrak{u}} \gamma_{j}\right] K_{\mathfrak{u}}(x, y) \quad \text { for all } x, y \in[0,1]^{d},
$$

where

$$
K_{\mathfrak{u}}(x, y)=\prod_{j \in \mathfrak{u}} \min \left(x_{j}, y_{j}\right) \quad \text { for all } x, y \in[0,1]^{d} .
$$


We stress that for all $\mathfrak{u}$, the function $K_{\mathfrak{u}}$ is a reproducing kernel and therefore

$$
\sum_{i, j=1}^{n} a_{i} a_{j} K_{\mathfrak{u}}\left(t_{i}, t_{j}\right) \geq 0 \text { for all } a_{j}, t_{j} \text { and } n \text {. }
$$

Furthermore, $\prod_{j \notin u} \gamma_{j} \leq 1$. This implies that

$$
\begin{aligned}
\frac{1}{\prod_{j=1}^{d} \gamma_{j}} & \sum_{i, j=1}^{n} a_{i} a_{j} K_{d, \gamma}\left(t_{i}, t_{j}\right) \\
& =\sum_{\mathfrak{u} \subseteq\{1,2, \ldots, d\}} \frac{1}{\prod_{j \notin \mathfrak{u}} \gamma_{j}} \sum_{i, j=1}^{n} a_{i} a_{j} K_{\mathfrak{u}}\left(t_{i}, t_{j}\right) \\
& \geq \sum_{\mathfrak{u} \subseteq\{1,2, \ldots, d\}} \sum_{i, j=1}^{n} a_{i} a_{j} K_{\mathfrak{u}}\left(t_{i}, t_{j}\right)=\sum_{i, j=1}^{n} a_{i} a_{j} \sum_{\mathfrak{u} \subseteq\{1,2, \ldots, d\}} K_{\mathfrak{u}}\left(t_{i}, t_{j}\right) \\
& =\sum_{i, j=1}^{n} a_{i} a_{j} \prod_{j=1}^{d}\left(1+\min \left(t_{i}, t_{j}\right)\right)=\sum_{i, j=1}^{n} a_{i} a_{j} K_{d}\left(t_{i}, t_{j}\right) .
\end{aligned}
$$

This proves that

$$
\frac{\left[e^{\text {wor }}\left(A_{n}\right)\right]^{2}}{\left[e^{\text {wor }}(0)\right]^{2}} \geq \frac{\left\|h_{d}\right\|_{H_{d}}^{2}-2 \sum_{j=1}^{n} a_{j} h_{d}\left(t_{j}\right)+\sum_{i, j=1}^{n} a_{i} a_{j} K_{d}\left(t_{i}, t_{j}\right)}{\left\|h_{d}\right\|_{H_{d}}^{2}} .
$$

The right-hand side is just the ratio of the square of the worst case error of $A_{n}$ and the square of the initial error for the unweighted case, i.e., for the space $H_{d}$. We can now apply Theorem 1 , which states that we have the curse of dimensionality for all nontrivial problems. In our case, $h_{1}(0)=0$ and $h_{1} \neq 0$ imply that the problem is indeed nontrivial. This completes the proof.

In view of Theorem 2 , we must assume that $h_{1}(0) \neq 0$. Is it enough to claim tractability results for fast decaying product weights? Not yet, since we have the second troublesome case. Namely, even for $d=1$ we may have exponential dependence on $\varepsilon^{-1}$. Indeed, as in Example 10.4.3 of Chapter 10 of $[5]$, for $j=1,2, \ldots$ define

$g_{j}(x)= \begin{cases}0 & \text { for } x \in[0,1 /(j+1)] \cup[1 / j, 1], \\ \sqrt{j(j+1)}[-x+1 /(j+1)] & \text { for } x \in\left[1 /(j+1), \frac{1}{2}(1 /(j+1)+1 / j)\right], \\ \sqrt{j(j+1)}[x-1 / j] & \text { for } x \in\left[\frac{1}{2}(1 /(j+1)+1 / j), 1 / j\right] .\end{cases}$

The functions $g_{j}$ are piecewise linear, the support of $g_{j}$ is $[1 /(j+1), 1 / j]$ and these functions have disjoint supports. Then $H_{1}=H_{1, \gamma}$. Clearly, $g_{j} \in H_{1}$ and $\left\langle g_{i}, g_{j}\right\rangle_{H_{1}}=\delta_{i, j}$.

Define $I_{1}(f)=\left\langle f, h_{1}\right\rangle_{H_{1}}$ with

$$
h_{1}(x)=\sum_{j=1}^{\infty} \alpha_{j} g_{j}(x) \quad \text { for all } x \in[0,1] .
$$


The coefficients $\alpha_{j}$ are chosen such that $\alpha_{1} \geq \alpha_{2} \geq \cdots \geq 0$ and

$$
\left\|h_{1}\right\|_{H_{1}}^{2}=\sum_{j=1}^{\infty} \alpha_{j}^{2}=1 .
$$

It is proved in Example 10.4.3 of [5] that

$$
\inf _{A_{n}} e^{\text {wor }}\left(A_{n}\right)=\left[\sum_{j=n+1}^{\infty} \alpha_{j}^{2}\right]^{1 / 2} .
$$

Obviously, we can now define the coefficients $\alpha_{j}$ so that the information complexity is exponential in $\varepsilon^{-1}$. For instance, take

$$
\alpha_{j}=\left[\frac{1}{\ln ^{2}(j+e-1)}-\frac{1}{\ln ^{2}(j+e)}\right]^{1 / 2} .
$$

Then it is easy to check that

$$
n\left(\varepsilon, I_{1}\right)=\left\lceil\exp \left(\varepsilon^{-1}\right)-e\right\rceil .
$$

Therefore the problem is not even weakly tractable.

One may hope that the lack of weak tractability happens only for some linear functionals. Unfortunately, this is not the case. Let

$$
B=\left\{h_{1} \in H_{1} \mid n\left(\varepsilon, I_{1}\right)=\Theta\left(\exp \left(\varepsilon^{-1}\right)\right)\right\}
$$

be the set of representers for which we have exponential dependence on $\varepsilon^{-1}$ even for $d=1$. It turns out that the set $B$ is dense in $H_{1}$; see again Example 10.4.3 of [5].

This discussion means that if we want to obtain, say, polynomial tractability for linear tensor product problems for the weighted Sobolev space $H_{d, \gamma}$, then we have to assume that $h_{1}(0) \neq 0$ and that the information complexity for the univariate case must be polynomial in $\varepsilon^{-1}$. Hence, let us assume that for a given $h_{1}$ there exists a positive $s$ such that

$$
h_{1}(0) \neq 0 \quad \text { and } n\left(\varepsilon, I_{1}\right)=\mathcal{O}\left(\varepsilon^{-s}\right) \quad \text { for all } \varepsilon \in(0,1) .
$$

We are ready to prove the following theorem.

Theorem 3. Let $I_{\gamma}=\left\{I_{d, \gamma}\right\}$ be a linear tensor product problem defined over the weighted Sobolev space $H_{d, \gamma}$ with $h_{1}$ satisfying (5). Then the following statements hold.

- If there exists $p \geq s$, with $s$ satisfying (5), such that

$$
\sum_{j=1}^{\infty} \gamma_{j}^{p /(p+2)}<\infty
$$

then $I_{\gamma}$ is strongly polynomially tractable with an exponent at most $p$, i.e.,

$$
n\left(\varepsilon, I_{d, \gamma}\right)=\mathcal{O}\left(\varepsilon^{-p}\right) \quad \text { for all } \varepsilon \in(0,1) \text { and } d \in \mathbb{N}
$$

with the factor in the big $\mathcal{O}$ notation independent of $\varepsilon^{-1}$ and $d$. 
- If there exists $p \geq s$ such that

$$
\limsup _{d \rightarrow \infty} \frac{\left(\sum_{j=1}^{d} \gamma_{j}^{p /(p+2)}\right)^{(p+2) / p}}{\ln d}<\infty,
$$

then $I_{\gamma}$ is polynomially tractable.

- If there exists $p \geq s$ such that

$$
\lim _{d \rightarrow \infty} \frac{\left(\sum_{j=1}^{d} \gamma_{j}^{p /(p+2)}\right)^{(p+2) / p}}{d}=0
$$

then $I_{\gamma}$ is weakly tractable.

Proof. The weighted tensor product (WTP) algorithm was introduced for product weights in [9] as a generalization of the Smolyak/sparse grid algorithm; see [8]. The basic information on the WTP algorithm can be also found Chapter 15 of [5]. In particular, Theorem 15.21 of [5] states that the error bounds of the WTP algorithm yield that $I_{\gamma}$ is strongly polynomially, polynomially or weakly tractable if the product weights satisfied the conditions presented here.

Theorem 3 tells us that if the weights decay fast enough, we have various notions of tractability. It is natural to ask whether the conditions presented on the product weights are sharp. It turns out that they are sometimes "almost" sharp. Indeed, for some specific linear tensor product functionals we know necessary and sufficient conditions on product weights to obtain tractability results. To see this we turn our attention to multivariate integration which is probably the most important and the most studied linear tensor functional. So, we let

$$
I_{d}(f)=\int_{[0,1]^{d}} f(t) \mathrm{d} t \quad \text { for all } f \in H_{d, \gamma} .
$$

This corresponds to

$$
h_{d}(x)=\int_{[0,1]^{d}} K_{d, \gamma}(x, t) \mathrm{d} t=\prod_{j=1}^{d}\left(1+\gamma_{j}\left(x_{j}-\frac{1}{2} x_{j}^{2}\right)\right) \quad \text { for all } x \in[0,1]^{d} .
$$

The initial error is given by

$$
\left\|I_{d, \gamma}\right\|=\prod_{j=1}^{d}\left(1+\frac{1}{3} \gamma_{j}\right)^{1 / 2} .
$$

It was proved in [3] that multivariate integration is strongly polynomially tractable iff

and polynomially tractable iff

$$
\sum_{j=1}^{\infty} \gamma_{j}<\infty
$$

$$
\limsup _{d \rightarrow \infty} \frac{\sum_{j=1}^{d} \gamma_{j}}{\ln d}<\infty .
$$


It was proved in [1] that multivariate integration is weakly tractable iff

$$
\lim _{d \rightarrow \infty} \frac{\sum_{j=1}^{d} \gamma_{j}}{d}=0 .
$$

We now compare these conditions with the conditions presented in Theorem 3 by assuming that the product weights are of the form $\gamma_{j}=j^{-\beta}$. Then we have the following facts.

- Strong polynomial tractability in Theorem 3 holds for $\beta>1$. This condition is necessary for strong polynomial tractability of multivariate integration. In this case, the estimate in Theorem 3 is sharp.

- Polynomial tractability in Theorem 3 also requires that $\beta>1$. For multivariate integration we must assume that $\beta \geq 1$. In this case, there is not much difference and the estimate in Theorem 3 is almost sharp.

- Weak tractability in Theorem 3 again requires that $\beta>1$. However, multivariate integration is weakly tractable iff $\beta>0$. In this case, there is a gap and it is not clear what is the condition on $\beta$ to guarantee that all linear tensor product functionals satisfying (5) are weakly tractable.

\section{Acknowledgment}

The work of the second author was partially supported by the National Science Foundation. The authors thank Art G. Werschulz for valuable comments on their paper.

\section{References}

[1] M. Gnewuch and H. Woźniakowski, Generalized tractability for linear functionals. In: Monte Carlo and Quasi-Monte Carlo Methods 2006, A. Keller, S. Heinrich, H. Niederreiter (eds.), Springer, Berlin, 2008, 359-381.

[2] E. Novak, I. H. Sloan and H. Woźniakowski, Tractability of tensor product linear operators. J. Complexity 13, 387-418, 1997.

[3] E. Novak and H. Woźniakowski, Intractability results for integration and discrepancy, J. Complexity 17 (2001), 388-441.

[4] E. Novak and H. Woźniakowski, Tractability of Multivariate Problems. Volume I: Linear Information. European Math. Soc., Zürich, 2008.

[5] E. Novak and H. Woźniakowski, Tractability of Multivariate Problems, Volume II: Standard Information for Functionals, European Math. Soc., Zürich, to appear.

[6] E. Novak and H. Woźniakowski, Tractability of Multivariate Problems, Volume III: Standard Information for Operators. In progress.

[7] I. H. Sloan and H. Woźniakowski, When are quasi-Monte Carlo algorithms efficient for high dimensional integrals? J. Complexity 14 (1998), 1-33.

[8] S. A. Smolyak, Quadrature and interpolation formulas for tensor products of certain classes of functions. Dokl. Akad. Nauk SSSR 4 (1963), 240-243.

[9] G. W. Wasilkowski and H. Woźniakowski, Weighted tensor-product algorithms for linear multivariate problems. J. Complexity 15 (1999), 402-447. 
[10] H. Woźniakowski, Tractability and strong tractability of linear multivariate problems, J. Complexity 10 (1994), 96-128.

[11] H. Woźniakowski, Tractability and strong tractability of multivariate tensor product problems. J. Comput. Inform. 4 (1994), 1-19.

Erich Novak

Mathematisches Institut, Universität Jena

Ernst-Abbe-Platz 2, 07740 Jena, Germany

e-mail: erich.novak@.uni-jena.de

Henryk Woźniakowski

Department of Computer Science, Columbia University,

New York, NY 10027, USA

and

Institute of Applied Mathematics, University of Warsaw

ul. Banacha 2, 02-097 Warszawa, Poland

e-mail: henryk@cs.columbia.edu

Open Access This article is distributed under the terms of the Creative Commons Attribution Noncommercial License which permits any noncommercial use, distribution, and reproduction in any medium, provided the original author(s) and source are credited. 